\title{
CLASIFICACIÓN DE PERFILES DE LECTORES DE UN PERIÓDICO DIGITAL
}

\section{CLASSIFICATION OF READERS PROFILES OF A DIGITAL JOURNAL}

\author{
Enrique De La Hoz Domínguez ${ }^{1}$, Adel Mendoza Mendoza ${ }^{2}$, Hoolyana Ojeda De La Hoz ${ }^{3}$
}

${ }^{1}$ Estudiante del doctorado en Estadística e Investigación Operativa (Universitat Politècnica de Catalunya), Especialista en Estadística, Ingeniero industrial, e-mail: enrique.jose.de.la.hoz.domingu@estudiant.upc.edu; ${ }^{2}$ Magíster en Ingeniería Industrial, Docente de Planta Programa de Ingeniería Industrial. Universidad del Atlántico, e-mail: adelmendoza@mail.uniatlantico.edu.co; ${ }^{3}$ Máster en comunicaciones digitales y nuevas tecnologías, e-mail: hojeda@uao.es

Rev. U.D.C.A Act. \& Div. Cient. 20(2): 469-478, Julio-Diciembre, 2017

\section{RESUMEN}

En este artículo de investigación, se analizó el comportamiento de lectores de un periódico digital, con el objetivo de encontrar los perfiles asociados al uso del sitio Web, con el propósito de definir estrategias de contenidos y nuevos productos del periódico. Se tomó una muestra de 689 usuarios, correspondiente a tres meses de actividad, a través de la herramienta Google Analytics; se determinaron las secciones del diario que se utilizaban en cada visita por los usuarios, para consolidar así la base de datos. En el proceso de análisis, se utilizaron las técnicas multivariadas, análisis de componentes principales y análisis de clúster, por medio, de la cual, se lograron gráficos y visualizaciones, que permiten una mejor interpretación de los resultados y ayudan al proceso de creación de perfiles de usuario. El resultado muestra la identificación de tres perfiles de usuario, para los cuales, se han definido estrategias de contenidos y de nuevos productos.

Palabras clave: Perfil de usuario, análisis multivariado, análisis de perfil, diario digital

\section{SUMMARY}

In this article, we analyzed the behavior of readers of a digital newspaper in order to find the profiles associated with the use of the website, in order to define content strategies and new newspaper products. A sample of 689 users was taken corresponding to three months of activity, through the tool Google Analytics, it was determined the sections of the newspaper that were used in each visit by the users to consolidate the database. In the process of analysis, the multivariate techniques are used main component analysis and cluster analysis, by means of which graphs and visualizations were obtained that allow a better interpretation of the results and help to the process of creation of user profiles. The result shows the identification of three user profiles, for which content strategies and new products have been defined.

Key words: User profiles, multivariate analysis, profile analysis, digital newspaper

\section{INTRODUCCIÓN}

Internet ha revolucionado cada uno de los aspectos de la vida diaria, hasta convertirse en una herramienta fundamental e, incluso, imprescindible, en nuestro día a día. Para el 2015, cerca del 50\% de la población mundial tenía acceso a Internet y en algunas regiones geográficas, como Europa, Norteamérica y Oceanía, tres de cuatro personas son usuarios frecuentes (Banco Mundial, 2016).

Esta revolución tecnológica abre un espacio para el desarrollo de nuevas oportunidades de negocio, crear nuevos canales informativos y promover la participación ciudadana en los espacios de toma de decisiones. Según los datos recogidos, mediante encuestas sobre los contenidos digitales, realizada por la firma Accenture, se revela cómo el $100 \%$ de los CEO encuestados expresan que los contenidos digitales son valiosos para los negocios y que el $73 \%$ de las firmas ha gastado más de 50 millones de dólares en contenidos, cada año (Accenture Digital, 2015).

Es así, como los medios de comunicación digitales deben adaptar sus contenidos, con base al nivel de conocimiento que tengan sobre el público objetivo, al cual, va dirigida su información, teniendo en cuenta que el internauta dejó de ser un simple consumidor pasivo y se convirtió en un 
protagonista activo, que controla, filtra, censura y distribuye, los contenidos que recibe. El usuario empoderado por las redes sociales decide cuándo, cómo y qué información necesita, accediendo a contenidos que les parezca oportunos, en términos de tiempo, de modo y de cantidad. El mercado de medios de información digital es altamente competitivo, encontrándose desde corporaciones, con músculos financieros muy grandes, hasta pequeños portales Web, con una visión "glocal" de la información, que se da al relacionar los elementos locales y particulares con los mundializados (Bolívar Botía, 2001). Durante el último cuarto de siglo, el uso comercial de Internet ha provocado que el panorama empresarial haya cambiado a un ritmo frenético; es así, como en el proceso de estrategia de marketing, donde las tecnologías digitales están teniendo y tendrá un impacto significativo (Kannan, 2017).

El periódico es uno de los más antiguos medios de comunicación y se ha considerado, durante mucho tiempo, como la principal forma de entrega de información (Chung, 2008). Con la llegada del Internet, se dio el nacimiento de un nuevo formato de presentación de los medios de comunicación, generando un periodismo distinto. En sus inicios, los medios de comunicación no daban mucho valor a su versión digital; ahora, el concepto de Web First (web primero) es imprescindible y consiste en que todos los contenidos se tratan antes en la Web que en el papel. Es más, algunos han nacido digitalmente, por ejemplo, Vilaweb y El Nacional, en España (López, 2015).

En primera instancia, las empresas periodísticas tradicionales visualizaron Internet como un nuevo canal de distribución. "Los periódicos tradicionales saben que hay que estar en la red, pero no saben muy bien para qué" (López \& Neira, 2000). La transformación fue tan rápida, que muchos periódicos no sobrevivieron a la etapa digital, solo aquellos que supieron acoplar el modelo de negocio en físico, a las oportunidades de la era digital. Es así, como surgen nuevos servicios de publicidad, de trazabilidad de usuarios, de personalización de la información y de interacción multimedia, que generan nuevas características a la definición de un periódico digital. En este tipo de formato, los usuarios participan en los cambios y en la evolución de estos sitios, porque son productores y revisores de los contenidos (Massari, 2010).

Para los periódicos digitales resulta muy importante conocer su público objetivo, identificando el perfil de sus lectores. El concepto de perfil de usuario de un lector, se puede definir como un vector $n$-dimensional, donde $n$ es el número de campos de cada característica para identificar un perfil (Vosecky et al. 2009). Estudios recientes han analizado las características de los usuarios de sitios Web: Ditoiu \& Platon (2012) describen los criterios que un consumidor considera importante en la publicidad de paquetes turísticos, teniendo en cuenta sus condiciones sociodemográficas; De Amo et al. (2015) proponen un método automático de un sistema de soporte de decisión de preferencias, basado en técnicas de minería, que consiste en extraer un perfil de usuario de un conjunto de muestras de preferencia de usuario; en el trabajo de Peng et al. (2016), se realiza una revisión de la literatura sobre sistemas de detección y de prevención de intrusiones, basados en perfiles de usuario, para confirmar la legitimidad de acceso en el sistema y Van Dam \& Van de Velden (2015) desarrollaron una metodología para explorar perfiles de usuarios de personas conectadas al sitio de Facebook de una empresa.

Conocer el comportamiento de los usuarios ayudará a los medios de comunicación que operan en Internet a identificar, de manera objetiva, su público objetivo, así como profundizar en el conocimiento de su caracterización, comportamiento digital, actitudes y opiniones, perfil socio-económico y los canales habituales de información. Esta información es vital para generar estrategias de posicionamiento y garantizar la sostenibilidad, en el largo plazo, como empresa.

Por lo anterior, el objetivo del presente trabajo está centrado en el análisis del comportamiento de los lectores de un periódico digital, en función del uso que hacen de éste, asociado a variables de horario, de frecuencia, de dispositivo de contacto, entre otras.

\section{MATERIALES Y MÉTODOS}

El enfoque metodológico, para la realización de la investigación, está basado en un estudio descriptivo, cualitativo y cuantitativo, para la identificación de perfiles de usuarios. Se utilizaron las técnicas multivariantes análisis de clúster y análisis de componentes principales. El análisis de clúster es una técnica que permite organizar la información de variables en grupos homogéneos, en función de su similitud o disimilitud entre ellos y el análisis de componentes principales (PCA) es un método multivariado, que permite estudiar y explorar un conjunto de datos de variables cuantitativas, medidas en un conjunto de objetos u observaciones (González, 2006). Dentro de las muchas aplicaciones de estas técnicas, se pueden destacar las que han sido empleadas para determinar características de usuarios (Granell et al. 2015; Al Wakeel \& Wu, 2016; Assimakopoulos, 2013; Morchid et al. 2014).

Los datos analizados fueron obtenidos del Google Analytics; para el estudio, se establecieron tres meses, como periodo de estudio, comprendidos entre el primero (1) de agosto y el treinta y uno (31) de octubre de 2016; en este lapso, se representa el comportamiento normal de los usuarios del diario. Considerando que la capacidad de Google Analytics de asignar un número de usuario único a cada visitante a la 
página es posible, se contó con la información, donde se establecieron las páginas que visitó el usuario, en el tiempo de estudio.

Para identificar los patrones o perfiles de comportamiento de los usuarios, se han definido las variables de segmentación y se ha realizado el seguimiento durante un periodo de tiempo de tres meses.

Las variables de segmentación consideradas fueron:

Portada (p): 1/0 si/no ha ingresado a la portada del diario (con umbral de tiempo mínimo).

Política y Sociedad (pys): 1/0 si/no configura una visita a la sección de política.

Economía (eco): 1/0 si/no configura una visita a la sección de economía.

Deportes (dep): 1/0 si/no configura una visita a la sección de deportes.

Cultura (cul): 1/0 si/no configura una visita a la sección de cultura.

Entrevistas (ent): 1/0 si/no configura una visita a la sección de entrevista.

Opinión (opi): 1/0 si/no configura una visita a la sección de opinión.

Videos (vid): $1 / 0$ si/no accede a la sección de videos.

Internacional (int): $1 / 0$ si/no accede a la sección internacional.

El promedio de actividad/uso de productos o servicios en cada categoría, se han utilizado para definir los perfiles de comportamiento estandarizados (independiente del número de visitas). El resultado promedio es el valor relevante de nuestro estudio, dado que para la creación de perfiles iniciales de usuarios es más importante la frecuencia que la intensidad. Los resultados obtenidos en una selección de usuarios, quienes han visitado el portal más de seis veces en tres meses, se organizaron en una base de datos.

\section{RESULTADOS Y DISCUSIÓN}

Análisis de correlación: Como fase preliminar al desarrollo de los perfiles, se analizó el nivel de correlación de las variables (Tabla 1), que permitió identificar qué secciones son visitadas, de manera frecuente, de manera conjunta. Los resultados muestran una fuerte correlación entre las secciones:

a. Portada, economía, deporte y cultura

b. Entrevista, opinión e internacional

c. Política y videos

Estos resultados preliminares dieron una idea del comportamiento de los usuarios en la interacción con el portal, que servirá como insumo para el desarrollo de los perfiles de usuario, objetivo principal del presente estudio.

Análisis de variables activas con componentes principales: Para aplicar el análisis de componentes principales, se utilizaron las secciones del diario y la interacción de los usuarios como variables; además, se incluyeron variables demográficas, que no actuaron como variables activas, pero ayudaron en la interpretación de los resultados.

Es así, como en el mapa de los ejes principales, se logra explicar un $80 \%$ de la inercia total (Figura 1). Cada uno de los puntos en el mapa corresponde a la representación

Tabla 1. Correlación de variables.

\begin{tabular}{|c|c|c|c|c|c|c|c|c|c|}
\hline & Portada & Política & Economía & Deportes & Cultura & Entrevista & Opinión & $\begin{array}{c}\text { Interna- } \\
\text { cional }\end{array}$ & Videos \\
\hline Portada & 1 & $-0,2$ & 0,8 & 0,8 & 0,8 & 0,3 & 0,4 & 0,3 & $-0,2$ \\
\hline Política & $-0,2$ & 1 & $-0,2$ & $-0,2$ & $-0,2$ & 0,4 & 0,3 & 0,4 & 0,9 \\
\hline Economía & 0,8 & $-0,2$ & 1 & 0,8 & 0,8 & 0,3 & 0,4 & 0,3 & $-0,2$ \\
\hline Deportes & 0,8 & $-0,2$ & 0,8 & 1 & 0,8 & 0,3 & 0,4 & 0,4 & $-0,2$ \\
\hline Cultura & 0,8 & $-0,2$ & 0,8 & 0,8 & 1 & 0,3 & 0,4 & 0,4 & $-0,2$ \\
\hline Entrevista & 0,3 & 0,4 & 0,3 & 0,3 & 0,3 & 1 & 0,7 & 0,7 & 0,5 \\
\hline Opinión & 0,4 & 0,3 & 0,4 & 0,4 & 0,4 & 0,7 & 1 & 0,8 & 0,4 \\
\hline Internacional & 0,3 & 0,4 & 0,3 & 0,4 & 0,4 & 0,7 & 0,8 & 1 & 0,6 \\
\hline Videos & $-0,2$ & 0,9 & $-0,2$ & $-0,2$ & $-0,2$ & 0,5 & 0,4 & 0,6 & 1 \\
\hline
\end{tabular}

Fuente: Elaboración propia. 
del usuario, según el peso específico de éste con los ejes principales; dado que existe una cantidad de datos considerables, esta aproximación visual permite identificar, inicialmente, los comportamientos generales de los usuarios.

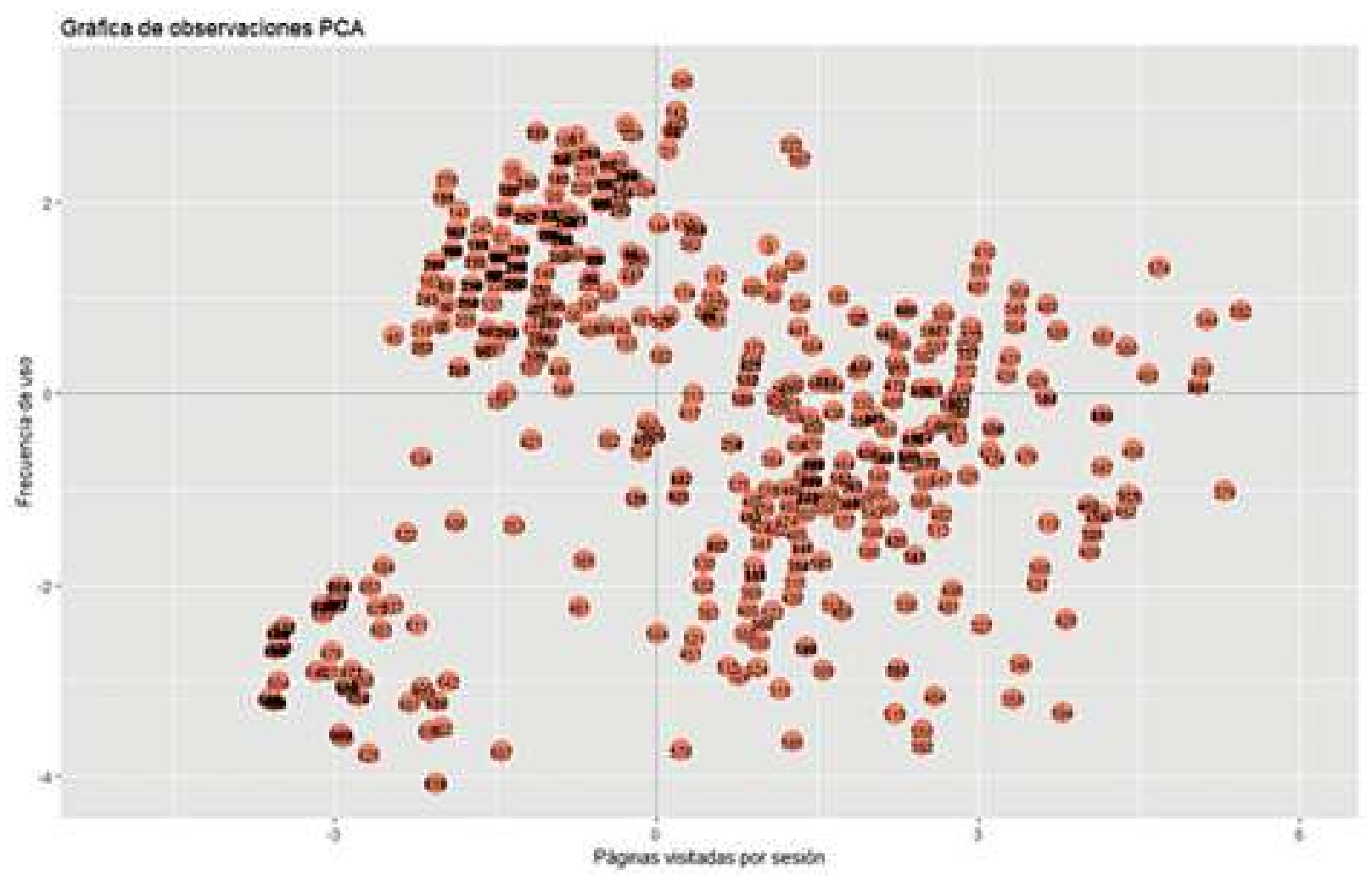

Figura 1. Mapa de lectores PCA.

El primer eje explica un $47 \%$ de la inercia, mientras que el segundo, un $33,3 \%$; claramente, se observa una concentración de usuarios en el centro de los ejes, muy común en este tipo de análisis (Peña, 2002). El eje horizontal posiciona a la derecha frecuencias elevadas de uso de las secciones de política, internacional, opinión y deportes; en el lado izquierdo del eje, se concentran los usuarios indicativos de "no uso" o del "uso poco frecuente". Por su parte, en la franja superior del eje vertical, se sitúan los usuarios asiduos de las secciones de política y de videos, en contraste con la parte inferior del eje, donde se ubican los usuarios menos asiduos. De esta manera, los datos muestran un orden natural ajustado a la escala, desde los usuarios poco frecuentes, hasta los más asiduos.

De cara a profundizar en el análisis, se representan algunas de las variables activas en diferentes planos, lo que facilitará la lectura de los resultados (Figura 2). Los puntos que se sitúen más a la derecha del plano estarán más relacionados con una interacción mayor de las secciones del diario. En este sentido, se observa que:

- Los dispositivos más minoritarios, móviles y tabletas resultan, a su vez, los más relacionados con usuarios intensivos de videos. El uso del Smartphone, se sitúa más a la derecha que el ordenador, denotando, también, una mayor relación con aquellos individuos que se conectan más a menudo.

- El momento del día también marca diferencias; los momentos de primera hora de la mañana o última del día tienden a estar más a la derecha del plano, relacionados con usuarios frecuentes de noticias internacionales, portada y opinión.

- Por otra parte, la edad en el uso parece no guardar relación con un uso intensivo de las secciones del periódico, ya que no encontramos un patrón claro de esta variable, en cuanto a su disposición en el plano. 


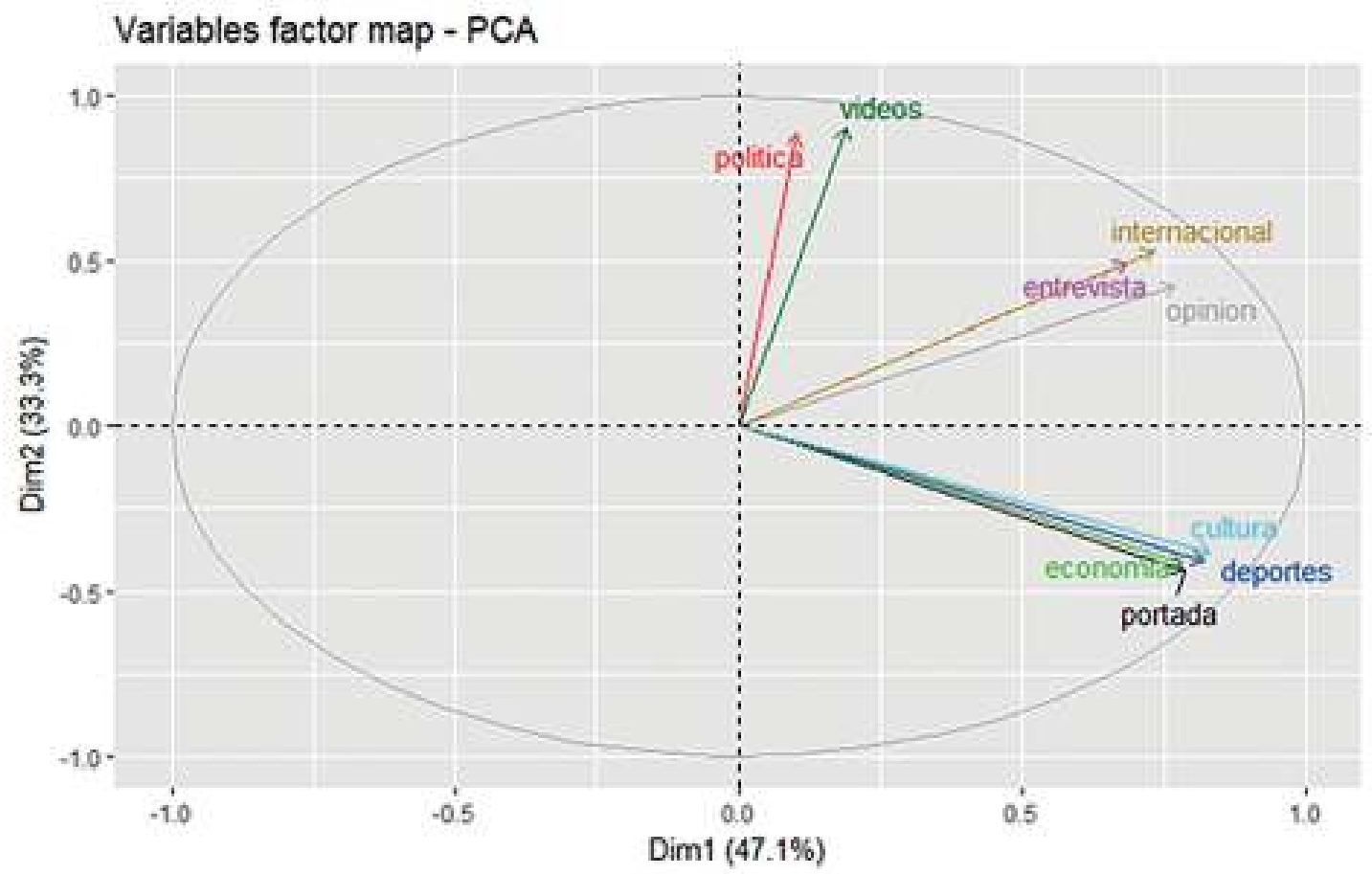

Figura 2. Espacio reducido PCA de variables.

Número de componentes principales a retener para el clúster: Para la aplicación del análisis clúster, se partió de la matriz de coordenadas principales de las observaciones, obtenidas a través del análisis de componentes principales. Como se indicó, se presentan varios criterios que pueden ayudar a determinar el número de componentes a retener y aquellos que servirán para el análisis posterior.

En este caso, el método del codo parecía indicar la conveniencia de retener dos componentes, explicando un alto porcentaje de la inercia inicial, en concreto, un 70,3\%; siguiendo, para esto, el criterio que recomienda retener un $60 \%$ de la inercia inicial, indica que los dos componentes principales son suficientes.

En la práctica, se probó con dos y tres componentes principales. En cualquier caso, dada la elevada dimensionalidad de los datos y con base en los resultados obtenidos, se consideró que la calidad de la información retenida es suficiente, para definir grupos bien perfilados.

Una vez decidido el número de ejes a retener, en el siguiente paso, se buscó determinar el número de segmentos a contemplar en la solución clúster. Para ello, se aplicaron diferentes criterios, en función de método clúster utilizado.
Determinación del número de clústeres en el análisis de clúster no jerárquico: El análisis jerárquico, se obtuvo aplicando el método de Ward, uniendo, en cada etapa, los dos clústeres, con el fin de obtener el menor incremento en el valor total de la suma de los cuadrados de las diferencias, dentro de cada clúster (Gallardo, 2011), como se muestra en la figura 3. En este gráfico, se dibujan, claramente, entre dos o tres grupos, evidenciando una estructura de agrupación. Se ha graficado el valor de la variabilidad intra-grupos, para ver cómo evoluciona dicho criterio, a medida que se pide un mayor número de perfiles a la solución clúster; el criterio siempre va a ser menor, a medida que aumenta el número de grupos. Para la determinación del número de grupos, se buscan cambios en la tendencia de la curva. El gráfico muestra un cambio de tendencia en el 2 y parece mostrar uno más ligero en la solución de 6 grupos.

Selección de la solución de clúster: Los resultados previos de los distintos métodos aplicados parecen apuntar a la existencia de 2 o 3 grupos. Dado el objetivo de este análisis, encontrar perfiles de lectores del diario y teniendo en cuenta lo heterogéneo que este ámbito puede ser fue que se decantó por las soluciones de tres grupos, dado que se buscó un poco más de detalle en los resultados, más allá de los 2 grandes grupos de usuarios, que puedan dibujarse. 


\section{Dendograma}

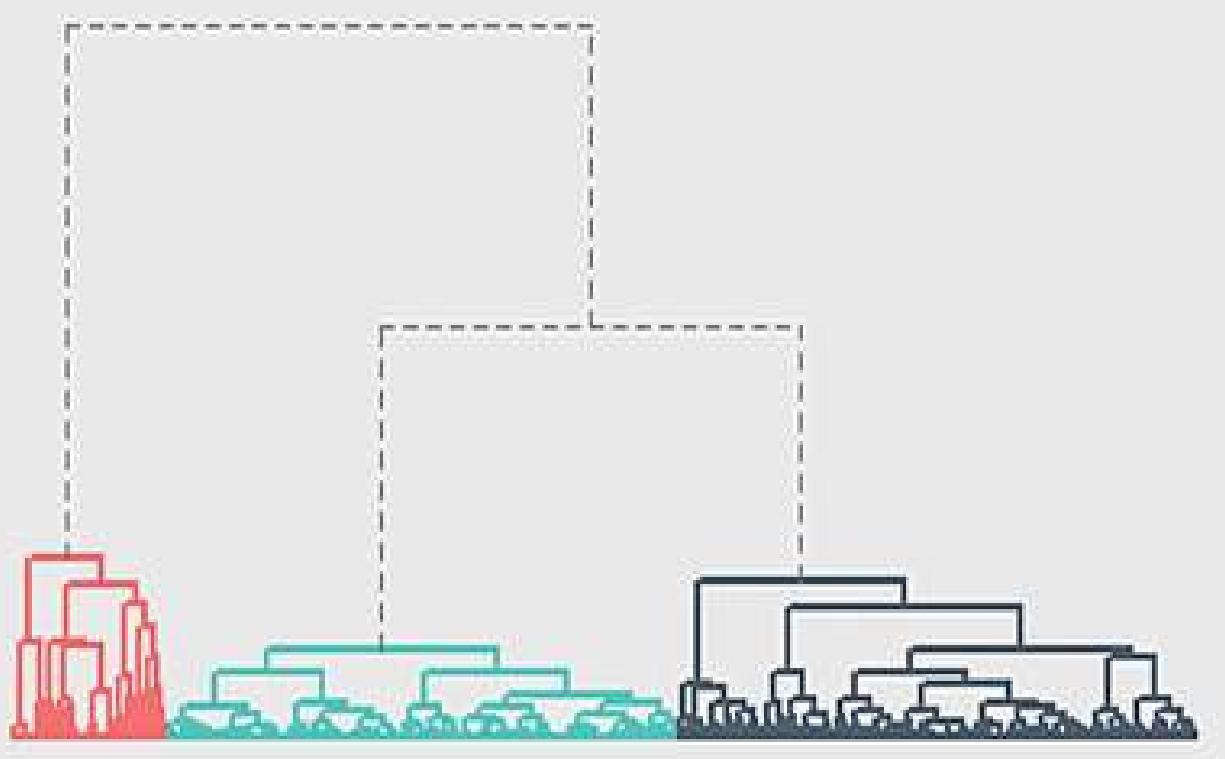

Figura 3. Dendograma representativo de los clústeres.

Visualización de los Perfiles creados: En el proceso de encontrar clústeres, se detectó que tres grupos se encuentran claramente identificados; en la figura 4, se aprecia el clúster de color verde, que ocupa mayoritariamente la parte derecha del plano, mientras el grupo de color rosa, se ubica en la parte superior izquierda y, por último, el azul, en la parte inferior izquierda.

\section{Definición de los perfiles identificados en el Análisis de Clúster:}

Perfil de lector 1 . El perfil de este lector es uno que responde a un comportamiento de consumo de información rápida; prefiere el formato de video, al cual, accede el $85,41 \%$ de las visitas; los contenidos que más consume son videos y política; tiene poco interés en temas de deportes y cultura. Se observa que solo un $8,69 \%$ ingresa a través de la portada, lo que indica que, posiblemente, interactúe mucho con el diario, por las redes sociales y foros.

Este perfil se denominó Visual, por su alta utilización de la sección de videos y poca relación con los contenidos de lectura. Este grupo representa el 36,3\% del total de la muestra a de usuarios, utilizada para este estudio. Se podría asemejar este perfil al de los nativos digitales, aquellos que prefieren los gráficos a los textos; utilizan mucho los accesos directos externos y comparten información con sus amigos, en forma permanente, en redes sociales (Hernández et al. 2014).
Perfil de lector 2. El perfil de este lector es uno que responde a un comportamiento clásico de lector de diarios, dado que utiliza la portada como ventana de ingreso, en un $30 \%$ de las veces y hace un recorrido por todas las secciones del diario, de manera sostenida. Muestra su mayor interés en las secciones de Opinión, Internacional y entrevistas, lo cual, da indicios de un lector que le interesa estar informado de la actualidad y se infiere que tienen al periódico, objeto de estudio, como uno de sus referentes de información.

Este perfil, se llama Informado, por su uso generalizado de las secciones del diario, mostrar un interés global y estar al día de lo que sucede en su entorno; este grupo representa el $50,5 \%$ del total de la muestra a, de usuarios utilizada para este estudio. Se puede asemejar este perfil al de los Inmigrantes digitales, que prefieren los procesos secuenciales, tuvieron que aprender un nuevo lenguaje, una nueva cultura y una nueva forma de comunicación; es como si aprendieran un nuevo idioma (Hernández et al. 2014).

Perfil de lector 3 . Es uno que responde a un comportamiento de poco interés en los contenidos del diario; en sus visitas al portal, casi no interacciona con las otras secciones; su ingreso al portal se apreció que es por medios externos, como redes sociales o foros, lo cual, muestra que, en momento, se sintió atraído por la información, pero si están en la Web, sale inmediatamente. Según Hernández et al. (2014), se podría 


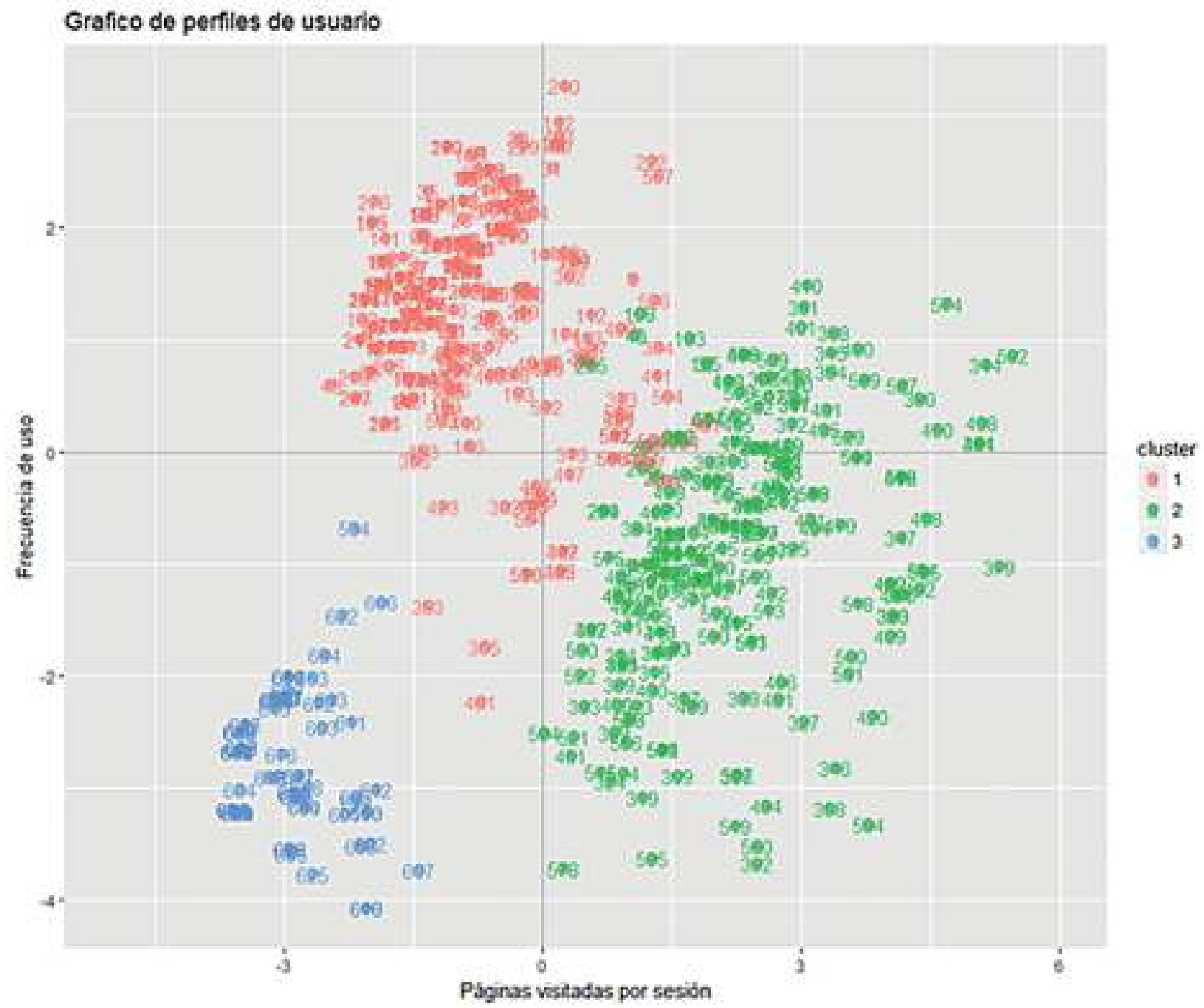

Figura 4. Resultado PCA con dos dimensiones.

asemejar este perfil al de visitante lurker (o husmeador), por ser un participante silencioso, con una actividad pasiva más que participativa; está ahí leyendo, observando los mensajes en los foros, acechando, pero de ninguna manera contribuye ni aporta a la discusión generada.

Este perfil, se llama Ni-ni (término asociado a personas que ni estudian, ni trabajan, equivalente al término en idioma inglés Neet, Not in employment or training, usado como analogía para la definición del perfil), por su poco uso del diario y su poca incidencia en el consumo de los contenidos, sin dejar de ser un grupo importante. Estos son los llamados no clientes, a los cuales, se les debe diseñar una oferta informativa diferente, dado que son los más cercanos en convertirse en usuarios recurrentes del portal, en un futuro (Palfrey \& Gasser, 2008). Este grupo representa el 13,2\% del total de la muestra a, de usuarios utilizada para este estudio.

\section{Descripción de la oferta informativa asociada a los perfiles de usuario}

Perfil visual: Dado que la sección más visitada por este grupo son los videos y la política, se debe promover la publicación de contenidos, que mezclen estos conceptos; en la actualidad, cada vez más se convierten en virales los videos de corte político, incluso, aquellos creados de manera oficial por los partidos políticos y el gobierno de turno. En función del diseño del portal Web sería una opción el pensar en ubicar, estratégicamente, estas dos secciones, una cerca de la otra, inclusive, inventar un producto especial, que enmarque estas dos categorías. Crear noticias basadas en infografías digitales, donde se presente contenido interactivo, que permita elevar la experiencia del usuario, haciéndolo participe del contenido. 
Perfil informado: El comportamiento clásico de este grupo hace que, en lo posible, se deba mantener una oferta informativa integral, con mucho contenido de actualidad, dado que, por lo general, hace un recorrido por todas las secciones, en busca de estar bien informado. Al ser lectores que replican los comportamientos del mundo físico en lo virtual, se puede pensar en tratar de generar un contacto físico con este usuario, a través de una carta, postal o una tarjeta de navidad.

Perfil Ni-ni: El comportamiento poco estructurado de este grupo plantea un reto en pos de crear una oferta informativa afín, dado que se observa un mínimo interés en temas de política, por lo cual, se podría generar ofertas innovadoras, en este campo.

En el presente trabajo, se logró encontrar perfiles de los diferentes lectores de un periódico digital (Tabla 2), con base en los datos recogidos a través del Google Analytics. El proceso empleado ha permitido, en una primera fase, la consideración de las nueve secciones principales del diario, tratando de replicar la diversidad de rutas que puede seguir un usuario, al momento de visitar el portal Web.
El uso del análisis multivariado permitió obtener una representación visual del comportamiento de las secciones del diario en interacción con los lectores, permitiendo una interpretación conjunta, en la cual, se observa cómo los usuarios que están interesados en videos, no muestran mayor interés por el resto de las secciones.

Un análisis de los perfiles muestra lo siguiente: los lectores del perfil informado suelen visitar, conjuntamente, las secciones de cultura, de deportes y de economía; además, son los que más hacen uso de la portada como guía de navegación, mientras que los usuarios del perfil Ni-ni son muy referenciados en la literatura, como Voyeristas digitales.

Los resultados obtenidos en los perfiles invitan a realizar estudios, en donde se involucren un mayor número de variables socioeconómicas, para poder proceder con una búsqueda exhaustiva en cada perfil; sin embargo, una de las restricciones de los diarios digitales pequeños es que no cuentan con gran cantidad de lectores registrados, por lo cual, se tendría que hacer uso de herramientas, como Google Analytics, que solo muestran información de manera

Tabla 2. Características de los perfiles.

\begin{tabular}{|c|c|c|c|}
\hline & Perfil de lector 1 & Perfil de lector 2 & Perfil de lector 3 \\
\hline Portada & $8,69 \%$ & $29,83 \%$ & $11,71 \%$ \\
\hline Política & $31,20 \%$ & $21,89 \%$ & $7,28 \%$ \\
\hline Economía & $0,00 \%$ & $11,16 \%$ & $1,16 \%$ \\
\hline Deportes & $1,77 \%$ & $12,47 \%$ & $1,54 \%$ \\
\hline Cultura & $2,33 \%$ & $13,15 \%$ & $1,43 \%$ \\
\hline Entrevista & $13,87 \%$ & $16,14 \%$ & $2,08 \%$ \\
\hline Opinión & $17,69 \%$ & $23,03 \%$ & $1,54 \%$ \\
\hline Internacional & $20,56 \%$ & $23,64 \%$ & $1,86 \%$ \\
\hline Videos & $85,41 \%$ & $29,98 \%$ & $3,68 \%$ \\
\hline
\end{tabular}

Fuente: Elaboración propia.

fragmentada y parcializada a sus intereses, dado que venden reportes automatizados, de comportamiento de usuario.

El proceso de recolección de datos fue la labor más ardua en el presente proyecto. De acuerdo con Zhang et al. (2016), se debe a la gran dificultad de filtrar manualmente cada pieza de información, para distinguir entre lo que más y menos les interesa a los usuarios, pero al final, se pudo cumplir el objetivo de obtener perfiles, bajo una estructura objetiva de análisis y la obtención de una representación gráfica, que puede ser muy útil a la hora de tomar decisiones.

Como punto de partida, para futuras investigaciones, se plantea la caracterización socioeconómica de los perfiles de usuario y la creación de herramientas de acceso abierto, para analizar el comportamiento de usuarios digitales. 
Conflicto de intereses: El manuscrito fue preparado y revisado con la participación de todos los autores, quienes declaramos que no existe conflicto de intereses, que ponga en riesgo la validez de los resultados presentados.

\section{BIBLIOGRAFÍA}

1. ACCENTURE DIGITAL .2015. State of Content Survey. Disponible desde Internet en: www.accenture. com/t20160322T055807_w__/us-en/_acnmedia/ Accenture/Conversion-Assets/DotCom/Documents/ Global/PDF/Digital_3/Accenture-Digital-ContentServices-Research-2015-Infographic.PDF (con acceso el 14/12/2016).

2. AL WAKEEL, A.; WU, J. 2016. K-means based cluster analysis of residential smart meter measurements. Energy Procedia. 88:754-760.

3. ASSIMAKOPOULOS, C. 2013. Mobile Internet users profile along with subscribers model of payment and attitudinal characteristics. Procedia Technology. 8:425-434.

4. BANCO MUNDIAL. 2016. Usuarios de internet. Disponible desde Internet en: http://datos. bancomundial.org/indicator/IT.NET.USER.P2 (con acceso el 15/01/2017).

5. BOLÍVAR BOTÍA, A. 2001. Globalización e identidades: (Des)territorialización de la cultura. Revista de Educación. 1:265-288.

6. CHUNG, D.S. 2008. Interactive features of online newspapers: Identifying patterns and predicting use of engaged readers. J. Computer $\square$ Mediated Communication. 13(3):658-679.

7. DE AMO, S.; DIALLO, M.S.; DIOP, C.T.; GIACOMETTI, A.; LI, D.; SOULET, A. 2015. Contextual preference mining for user profile construction. Information Systems. 49:182-199.

8. DITOIU, M.C.; PLATON, O.E. 2012. Aspects Regarding the Romanian Social Network User's Profile and its Implications in Marketing Destination Pages. Procedia Economics and Finance. 3:182-187.

9. GALLARDO, M. 2011. Métodos jerárquicos análisis de clúster. Disponible desde Internet en: www.ugr. es/ gallardo/pdf/cluster-3.pdf. (Con acceso el 22/02/2017).
10. GONZÁLEZ, C.G. 2006. Tratamiento de datos. Ediciones Díaz de Santos. 207p.

11. GRANELL, R.; AXON, C.J.; WALLOM, D.C. 2015. Clustering disaggregated load profiles using a Dirichlet process mixture model. Energy Conversion and Management. 92: 507-516.

12. HERNÁNDEZ, D.; RAMÍREZ, A.; CASSANY, D. 2014. Categorizando a los usuarios de sistemas digitales. Rev. Medios y Educación. 44:113-126.

13. KANNAN, P.K. 2017. Digital marketing: A framework, review and research agenda. International Journal of Research in Marketing. 34(1):22-45.

14. LÓPEZ, G. 2015. Periodismo digital. Redes, audiencias y modelos de negocio. Salamanca: Comunicación Social.

15. LÓPEZ GARCÍA, X.; NEIRA CRUZ, X.A. 2000. Los medios locales ante los desafíos de la red. Ámbitos. 4:101112.

16. MASSARI, L. 2010. Analysis of MySpace user profiles. Information Systems Frontiers. 12(4):361-367.

17. MORCHID, M.; DUFOUR, R.; BOUSQUET, P. M.; LINARES, G.; TORRES-MORENO, J.M. 2014. Feature selection using Principal Component Analysis for massive retweet detection. Pattern Recognition Letters. 49:33-39.

18. PALFREY, J.; GASSER, U. 2008. Born Digital: Understanding the first generation of digital natives. New York: Basic Books.141p.

19. PENG, J.; CHOO, K.K.R.; ASHMAN, H. 2016. User profiling in intrusion detection: A review. Journal of Network and Computer Applications. 72:14-27.

20. PEÑA, D. 2002. Análisis de Datos Multivariantes. Madrid: Mc Graw Hill.159p.

21. VAN DAM, J.W.; VAN DE VELDEN, M. 2015. Online profiling and clustering of Facebook users. Decision Support Systems. 70:60-72.

22. VOSECKY, J.; HONG, D.; SHEN, V.Y. 2009. User identification across multiple social networks. In Networked Digital Technologies, 2009. NDT'09. First International Conference on. p.360-365). IEEE. 
23. ZHANG, Z.; LIU, Y.; XU, G.; CHEN, H. 2016. A weighted adaptation method on learning user preference profile. Knowledge-Based Systems. 112:114-126.

Recibido: Febrero 21 de 2017

Aceptado: Octubre 14 de 2017

Cómo citar:

De La Hoz Domínguez, E.; Mendoza Mendoza, A.; Ojeda De La Hoz, H. 2017. Clasificación de perfiles de lectores de un periódico digital. Rev. U.D.C.A Act. \& Div. Cient. 20(2): 469-478. 